\title{
Near-Earth Object Characterization Priorities and Considerations for Planetary Defense
}

\author{
Paul A. Abell \\ 281-483-0293 (Office) \\ NASA Johnson Space Center (JSC) \\ paul.a.abell@nasa.gov
}

Carol Raymond (CalTech/Jet Propulsion Laboratory (JPL))

Terik Daly (Johns Hopkins University Applied Physics Laboratory (JHUAPL))

Daniel R. Adamo (Independent Astrodynamics Consultant)

Brent W. Barbee (NASA Goddard Space Flight Center (GSFC))

Megan Bruck Syal (Lawrence Livermore National Laboratory (LLNL)

Kyla Carte (George Mason University)

Nancy L. Chabot (Johns Hopkins University Applied Physics Laboratory (JHUAPL))

Jesse L. Dotson (NASA Ames Research Center (ARC))

Lee D. Graham (NASA Johnson Space Center (JSC))

Rob R. Landis (NASA Johnson Space Center (JSC))

Amy Mainzer (University of Arizona)

Mike C. Nolan (University of Arizona)

Edgard Rivera-Valentin (Lunar and Planetary Institute, USRA)

Andy S. Rivkin (Johns Hopkins University Applied Physics Laboratory (JHUAPL))

Patrick A. Taylor (Lunar and Planetary Institute, USRA)

Flaviane C. F. Venditti (Arecibo Observatory - University of Central Florida)

Lorien Wheeler (NASA Ames Research Center (ARC)) 


\section{Introduction}

Early detection and characterization of potentially hazardous near-Earth objects (NEOs) is the first priority for planetary defense as stated in the National Near-Earth Object Preparedness Strategy and Action Plan (NSTC 2018). Detailed knowledge of the orbital and physical properties of potentially hazardous NEOs, in addition to any associated uncertainties in these properties, is required to effectively plan and execute planetary defense mitigation activities. The orbital and physical characteristics of a NEO play a crucial role in determining the potential damage it could inflict and in predicting how the object may respond to any mitigation attempts. Such data can be obtained from a combination of remote observations from ground and space-based assets, as well as from launching spacecraft missions for in situ reconnaissance.

Current NEO survey efforts are focused on discovering as many objects as possible and have been successful in finding more than $90 \%$ of the objects larger than $1 \mathrm{~km}$ diameter. However, only approximately one third $(\sim 9,200)$ of the total estimated population of NEOs $\geq 140 \mathrm{~m}$ in diameter $(\sim 25,000)$ have been found to date. These objects are capable of causing extensive damage on regional or global scales resulting in extreme loss of life and property. Thus completing the NEO survey to discover the remaining $\sim 15,800$ NEOs within this size range has been recognized as a high priority since finding at least $90 \%$ of these objects reduces the overall impact risk by $>90 \%$ (Stokes et al., 2003; 2017). However, even NEOs < $140 \mathrm{~m}$ can cause significant damage and harm, albeit at smaller scales, as seen from evidence of the Tunguska 1908 and Chelyabinsk 2013 airburst events (Chyba et al., 1993; Popova et al., 2013; Mathias et al., 2017). Hence efforts to monitor and characterize these smaller NEOs are warranted whenever they are detected (NASEM 2019).

Discovery and tracking of NEOs is the first step in recognizing any potential threats of impact and facilitating appropriate action. If a hazardous object can be identified sufficiently far in advance, there is a higher likelihood that mitigation efforts will reduce the probability of impact, or even eliminate it entirely. Therefore maintaining current survey capabilities augmented by future NEO survey systems (e.g., Rubin Observatory and NEO Surveillance Mission (NEOSM)) that focus on discovering and identifying $>90 \%$ of the potentially hazardous NEOs $\geq 140 \mathrm{~m}$ in diameter (and a significant fraction of NEOs $<140 \mathrm{~m}$ ) are of the utmost priority in order to have sufficient warning time to plan, develop, test, and execute effective mitigation strategies (NASEM 2019; Barbee et al., 2020; Mainzer et al., 2020).

Once a potentially hazardous object is identified, subsequent characterization of the object is required in order to assess: whether or not the object poses an impact threat; the magnitude of the potential impact; and what mitigation technique(s) may be most appropriate to employ for the given situation. As stated above, gaining a better understanding of the orbital state of the NEO and its physical properties are the most important factors in assessing the threat from a potential impactor (NASEM 2019). However, in the early phases of discovery, these data may be relatively limited, leading to large uncertainties as to the true magnitude of the hazard associated with the object. Depending on the probability of impact, amount of warning time, and potential damage/loss of life, decisions related to the development of any action plan involving mitigation of the NEO may have to be made based on incomplete knowledge of the hazard. In a high-consequence event with relatively modest warning time, one method to reduce the uncertainty, and gain better understanding of the object's orbital and physical characteristics in a timely manner, would be to launch an in-situ reconnaissance mission. This additional characterization information would 
allow for a more complete understanding of the NEO's potential impact hazard, and, if warranted, enable a more effective and reliable mitigation plan to be implemented.

This paper outlines the NEO characterization priorities and considerations relevant to planetary defense over the time period of the Planetary Science and Astrobiology Decadal Survey 20232032. The following pages describe the most relevant NEO properties for assessing impact threats, identify which properties are required for planning an effective mitigation response, suggest how such properties can be most effectively determined, list properties that inform mitigation effectiveness, and recommend several activities to improve overall planetary defense readiness.

\section{Properties for Assessing Impact Threats}

There are several key properties that are relevant for assessing the overall impact threat from any known potentially hazardous NEO. In order of descending importance, these key properties are:

- Orbit elements and uncertainties - indicates the likelihood of impact, the impact velocity, the time of impact, and possible impact locations.

- $\quad \underline{\text { Size }}$ - acts as a proxy for mass.

- $\quad$ Spectral class - helps to further constrain mass and physical/chemical attributes.

- Evidence for multiple objects - raises the possibility of more than one impacting body.

Some knowledge of these properties is often obtained during the object's discovery apparition, but may not be complete enough to adequately assess impact probability depending on the initial observational circumstances and warning time. Usually this information is later refined somewhat as more observations of the NEO's characteristics are conducted, but there could be circumstances where the viewing geometry and/or distance of the object with respect to Earth prohibit detailed characterization, leading to relatively large uncertainties pertaining to impact hazards.

Knowledge of the NEO's orbital elements and their respective uncertainties is undoubtedly the most crucial property to understand since this determines the probability of impact, when the impact will occur, what the impact velocity will be, and where the impact location(s) will likely take place. The initial discovery observations can immediately identify if a particular NEO is an object of interest, and inform the NEO observing community and government decision makers as to the urgency of the situation. Subsequent observations of the object will help refine its orbital state and determine a more accurate orbit, which in turn will lead to more refined impact predictions. The size of the NEO is another key factor in assessing the impact hazard since it is a proxy for estimating the mass of the NEO. The estimated mass and velocity help bound the range of energies that may be associated with the NEO's impact, which indicate whether it will be relatively benign (i.e., detonate harmlessly in the upper atmosphere) or cause significant ground damage/loss of life at local, regional, or global scales.

Two other properties that are useful in assessing impact threats of potentially hazardous NEOs are spectral class and the presence of companion objects. Determination of the spectral class is used to infer whether the object may be rocky, metallic, anhydrous, or hydrous, and helps to link the object to meteorite analogs, which in turn constrain its density and mass. Spectral classification provides important information that feeds into the hazard models, which can be used to identify which objects are of interest and those that represent little to no danger (Reddy et al., 2019). In addition, any observational evidence to suggest that a potentially hazardous NEO may be a system 
of more than one object is also important to consider when trying to assess impact hazards given that a significant fraction of NEOs (about 1 in 6 larger than $200 \mathrm{~m}$ in diameter) exist in binary or ternary systems (Nolan et al., 2020; Virkki et al., 2020). Note that in some instances definitive detection of companion objects from remote observations may be difficult due to the orbital orientation of the system and/or the observational circumstances at the time of the NEO's apparition. However, it is plausible that in the case of a binary or ternary NEO on a possible impact trajectory with the Earth, both the primary and its companion(s) would be of sufficient size to cause concern.

These four key properties for assessing impact threats can be obtained using ground-based and space-based telescopes conducting remote observations of potentially hazardous NEOs over a variety of wavelengths. Collecting such information as early as possible through an assortment of techniques (i.e., broadband color photometry, spectroscopy, adaptive optics imaging, light curve rotational studies, radar astrometry and imaging, thermal IR photometry, etc.) enables a clearer understanding of the potential impact threat and allows for more informed decisions regarding subsequent planetary defense mitigation action plans (i.e., shelter in place, evacuation, spacecraft launch preparations, etc.). This initial characterization of the NEO would also allow for a wider range of responses to be considered for implementation, since early threat assessment enables multiple viable options for active mitigation (Barbee, 2020).

\section{Properties for Planning an Effective Response to a NEO Impact}

If a NEO presents a significant Earth impact probability, further characterization of the object is required in order to plan an effective mitigation response. The following properties for further detailed characterization are listed in relative priority order below:

- $\quad$ Precise orbit - indicates definitively whether or not an impact will occur, the specific location of the impact, and informs how much deflection is needed.

- $\underline{\text { Mass }}$ - determines the energy and momentum required for any deflection/disruption attempt.

- $\quad$ Presence of multiple objects - confirms directly how many objects are present and informs any special considerations required for deflection or disruption of a multiple body system.

- Detailed shape - aids the estimate of bulk density and porosity when the NEO's shape is combined with its mass, and informs how momentum may be transferred during a mitigation attempt.

- $\quad$ Material properties - constrains the magnitude of the momentum enhancement factor $(\beta)$ for any kinetic impactor deflection mitigation attempt. Properties such as strength and porosity are key factors that influence the NEO's response to mitigation deflection/disruption attempts (i.e., impact cratering and ejecta formation).

- Internal structure - influences the response of the NEO to the deflection/disruption attempt since NEOs may have a variety of internal structures (e.g., rubble pile vs. fractured shard vs. monolith) that will affect the outcome of such attempts. The internal structure may play a more significant role in the efficacy of disruption mitigation techniques.

- Mineral composition - helps with the selection of the specific deflection/disruption method since it affects nuclear mitigation attempts of objects comprised of volatile minerals or of those with significant iron content within the first few centimeters of the surface. A precise determination of composition appears to play no significant role in kinetic impactor mitigation efforts. 
- Detailed surface topology - assists modeling of how ejecta will behave and how much may be released from a mitigation attempt, which will affect the amount of deflection achieved. Impact site geology (e.g., boulders vs. fine grained regolith) can either enhance or reduce momentum transfer efficiency, depending on the specifics of the location. In addition, maps of surface characteristics can be used to assess the probable nature of the impact/detonation site for a deflection or disruption attempt and inform mission planning. These data also can be used to gain an understanding of the NEO's internal structure through analyses of boulder areal coverage, boulder size frequency distribution, and regolith characterization.

- $\quad$ Rotational state - affects the selection and magnitude of the mitigation approach since fastspinning objects may be harder to deflect for some methods. Additionally, NEOs with relatively fast rotation rates may have an increased likelihood of unintentional disruption, thus compromising the effectiveness of the mitigation.

- Dust/coma/ejecta - present a potential hazard to certain mitigation approaches if particle sizes are capable of interfering with spacecraft operations and deployment. Detection of any such particles informs special considerations that may be required for mitigation.

It should be noted the NEO population represents a diverse assemblage of objects, which has significant variation in terms of orbital dynamics, rotational state, physical properties (e.g., size, mass, shape, composition, internal structure, etc.), and near-field environment (e.g., dust, coma, ejecta, and companions) (Nolan et al., 2020). Continued discovery, tracking, and characterization of this population, both from remote sensing and in-situ observations, enables a more complete understanding of possible properties a potentially hazardous NEO may present (Barbee et al., 2020; Taylor et al., 2020). Although knowledge of these properties would be invaluable in the event of a definitive threat from a specific object, there is significant value in assessing the diversity of these properties across the NEO population to facilitate mitigation preparation. As noted by the National Academies, "Although the physical characteristics of an individual NEO that might strike Earth cannot be accurately predicted in advance, the knowledge of the range of possible characteristics will greatly aid in advance planning and might be essential if there is no opportunity to perform detailed characterization studies of the incoming NEO." (NRC, 2010).

\section{Determining NEO Properties Relevant to Mitigation}

In-situ characterization missions to potentially hazardous NEOs have provided major breakthroughs in understanding the physical properties of these objects, and have led to some new insights pertaining to the diversity among them (e.g., Hayabusa, Hayabusa2, and OSIRIS-REx). These missions relied heavily on remote sensing data to characterize their targets and prepare their mission operation plans prior to arrival. However, each of these missions encountered objects with physical properties (e.g., boulders instead of fined grained regolith) that were unexpected based on the best interpretations of the remote sensing data at the time, which impacted their operations. This demonstrates the limitations of remote sensing techniques to accurately determine physical properties, and highlights the utility of in situ observations to provide ground-truth data. In the interest of planetary defense, the National Academies report notes that, "The data collected from NEO characterization missions would also help to calibrate the ground- and space-based remote sensing data and may permit increased confidence in the remote classification of NEOs and their associated physical characteristics, which could inform future mitigation decisions." (NRC, 2010). 
Spacecraft reconnaissance missions are the most effective method for determining the properties of a NEO and reducing the uncertainties associated with a future impact scenario. These detailed properties can be determined via in-situ characterization by either rendezvous or flyby spacecraft depending on the amount of warning time prior to a potential impact, and assessment of the impact probability (NRC, 2010; Barbee, 2020). Each reconnaissance mission type has its own advantages and disadvantages. Flyby missions encounter the $\mathrm{NEO}$ at several $\mathrm{km} / \mathrm{s}$ and do not have the time for extensive examination of the object. In addition, flyby maneuvers are often conducted at some distance from the target, which limits determination of properties relevant for planetary defense consideration (e.g. mass, detailed surface topology, etc.). However, a reconnaissance flyby can give a rapid assessment of the NEO's nature and reduce key uncertainties related to properties that may not be adequately constrained from ground-based or space-based remote sensing observations (e.g., refined orbit, shape and size, presence of companions, etc.). For NEOs with relatively short warning times, utilization of flyby reconnaissance missions may be necessary to help inform an appropriate rapid planetary defense response.

In contrast, rendezvous missions have much more time to characterize the NEO and more accurately determine key properties most relevant to planetary defense implementation (e.g., mass, density, internal structure, composition, etc.). These missions also have the added capability to monitor the NEO over time, conduct close proximity observations, interact with the NEO by deploying surface assets/experiments (e.g., Hayabusa2's lander, rovers, and impactor), observe mitigation attempts, and then assess the effectiveness of the mitigation efforts. Utilization of a rendezvous mission in this manner gives an increased level of flexibility for impact threat assessment and mitigation planning. However, in spite of these advantages, rendezvous missions tend to not have as many launch opportunities, generally involve larger spacecraft, require more propellant, and tend to be more complex than flyby missions (Barbee, 2020). Rendezvous missions are well suited to situations where there is sufficient warning time to effectively determine the NEO's properties most pertinent for planetary defense considerations.

\section{In-Situ Characterization of NEO Properties for Assessing Mitigation Efforts}

There are a few specific physical properties of particular interest that should be characterized via in situ methods in order to understand the effectiveness of a fast-push NEO mitigation attempt (such as kinetic impact or nuclear detonation) and inform future mitigation efforts:

- High-resolution imaging of the impact or detonation site - The efficiency of the momentum transfer is affected by the terrain of the NEO in terms of the impact/detonation angle with respect to the local surface slope, the regolith geotechnical characteristics, and the presence of boulders at the target site.

- High-resolution determination of global shape and local topography of the impact or detonation site - The global shape and local topography can significantly affect how momentum is transferred. In addition, any shadowing of the surface by shape/topography features reduces the effects from a nuclear detonation.

- Accurate determination of the impact or detonation location relative to the NEO center-of$\underline{\text { mass }}$ - This affects how the impulse is partitioned into spin and translation components influencing the net change in the NEO's position.

- High-resolution observations of crater formation and ejecta production - Characterization of the mitigation crater's dimensions and measurement of the ejecta produced will indicate the efficiency of the mitigation and magnitude of the momentum enhancement factor $(\beta)$. 
NASA's DART and ESA's Hera missions are examples of in situ missions that will advance understanding of NEO physical properties that affect the efficiency of a kinetic impact deflection and inform future mitigation efforts. In late 2022, DART is scheduled to target and impact Dimorphos, the smaller companion in the (65803) Didymos binary NEO system. Hera will arrive in early 2027 to characterize both objects. The DART and Hera missions will provide the first detailed information on how a NEO larger than $140 \mathrm{~m}$ in diameter responds to a mitigation attempt.

\section{Recommended Activities to Improve Planetary Defense Readiness}

The following are recommended activities and missions to improve planetary defense readiness with respect to NEO characterization priorities for the next decade:

- Provide adequate and stable support for a robust Research and Analysis program (e.g., observations, experiments, theory, modeling, etc.) centered on NEO characterization and planetary defense mitigation.

- Maintain current survey capabilities and support the launch and operation of the NEO Surveillance Mission (NEOSM) to focus on discovering and identifying $>90 \%$ of the potentially hazardous NEOs $\geq 140 \mathrm{~m}$ in diameter.

- Continue detection and characterization of the NEO population to identify the range of physical properties that a potentially hazardous NEO may present, which would inform future planetary defense preparation and planning.

- Advance development of technologies relevant to NEO characterization particularly for in situ spacecraft missions (e.g., instruments/sensors, guidance navigation and control, etc.).

- Develop and launch in situ spacecraft missions to place previously obtained remote sensing measurements in context, and provide detailed characterization for NEO properties of interest that cannot be achieved from ground-based or space-based remote sensing (e.g., flyby tours of NEOs, rendezvous missions with assets for physical interaction, etc.).

- Hold additional exercises for early detection, characterization, and rapid assessment of potentially hazardous NEOs to test implementation of planetary defense protocols (e.g., International Asteroid Warning Network global observing campaigns for $2012 \mathrm{TC}_{4}$ and 66391 Moshup) (Reddy et al., 2019).

- Execute a rapid reconnaissance in situ mission (flyby or rendezvous) to a potentially hazardous NEO to demonstrate capabilities for planetary defense readiness (e.g., 99942 Apophis) (Barbee et al., 2020).

- Conduct a kinetic impact mitigation test on an appropriately sized benign NEO that has been previously characterized by a rendezvous spacecraft, which will remain on station to observe and monitor the effects of the impact deflection. This will provide additional data for determination of $\beta$ and extend the knowledge of NEO properties relevant to mitigation.

\section{Summary}

Early detection and characterization of a potentially hazardous NEO is of utmost importance to planetary defense. Obtaining detailed knowledge of the NEO's orbital and physical properties as soon as possible enables a better understanding of the potential impact consequences and uncertainties, which aids the preparation of the overall planetary defense response. This knowledge will help inform the need for: conducting in situ reconnaissance; modeling outcomes for possible mitigation attempt(s); preparing mitigation (deflection/disruption) efforts; performing cost/benefit analyses (e.g., in situ mitigation vs. accepting the impact); and planning appropriate civil defense responses for life and property. The recommended activities listed above will significantly improve 
planetary defense readiness in the years ahead and are submitted for consideration to the Planetary Science and Astrobiology Decadal Survey 2023-2032.

\section{References}

Barbee B. (2020). Near-Earth Object Characterization Priorities for Planetary Defense, $22^{\text {nd }}$ Small Bodies Assessment Group meeting, Pasadena, CA

Barbee B., et al., (2020). Future Spacecraft Missions for Planetary Defense Preparation. Whitepaper submitted to The Planetary Science and Astrobiology Decadal Survey 2023-2032.

Chyba, C. F., et al., (1993). The 1908 Tunguska explosion: atmospheric disruption of a stony asteroid, Nature 361, 40-44.

Mainzer, A., et al., (2020). The Future of Planetary Defense in the Era of Advanced Surveys. Whitepaper submitted to The Planetary Science and Astrobiology Decadal Survey 2023-2032.

Mathias, D. L., et al., (2017). A probabilistic asteroid impact risk model: assessment of sub300m impacts. Icarus 289, 106-119.

National Academies of Sciences, Engineering, and Medicine (NASEM), (2019). Finding Hazardous Asteroids Using Infrared and Visible Wavelength Telescopes. Washington, DC: The National Academies Press.

National Research Council (NRC) (2010). Defending Planet Earth: Near-Earth-Object Surveys and Hazard Mitigation Strategies. Washington, DC: The National Academies Press.

National Science and Technology Council (NSTC), (2018). National Near-Earth Object Preparedness Strategy and Action Plan: A Report by the Interagency Working Group for Detecting and Mitigating the Impact of Earth-Bound Near-Earth Objects of the National Science \& Technology Council. https://www.whitehouse.gov/wpcontent/uploads/2018/06/National-Near-Earth-ObjectPreparedness-Strategy-and-Action-Plan-23-pages-1MB.pdf.

Nolan, M., et al., (2020). Near-Earth Objects. Whitepaper submitted to The Planetary Science and Astrobiology Decadal Survey 2023-2032.

Popova, O. P., et al., (2013). Chelyabinsk Airburst, Damage Assessment, Meteorite Recovery, and Characterization, Science 342, no. 6162, 1069-1073.

Reddy, V., et al., (2019). Near-Earth asteroid 2012 TC 4 observing campaign: Results from a global planetary defense exercise. Icarus, 326, 133-150.

Stokes G., et al., (2003). A Study to Determine the Feasibility of Extending the Search for NearEarth Objects to Smaller Limiting Diameters. Report of the Near-Earth Object Science Definition Team, Prepared at the Request of National Aeronautics and Space Administration Office of Space Science Solar System Exploration Division. https://www.nasa.gov/sites/default/files/atoms/files/pdco-neoreport030825.pdf.

Stokes, G., et al., (2017). Update to Determine the Feasibility of Enhancing the Search and Characterization of NEOs. Report of the Near-Earth Object Science Definition Team, Prepared at the request of National Aeronautics and Space Administration, Science Mission Directorate, Planetary Science Division. https://www.nasa.gov/sites/default/files/atoms/files/2017_neo_sdt_final_e-version.pdf.

Taylor, P. A., et al., (2020). Ground-based Radar for Planetary Science and Planetary Defense. Whitepaper submitted to The Planetary Science and Astrobiology Decadal Survey 2023-2032.

Virkki, A., et al., (2020). Near-Earth Object Characterization Using Ground-based Radar Systems. Whitepaper submitted to The Planetary Science and Astrobiology Decadal Survey 2023-2032. 